\title{
Efficacy and safety of poly-D,L-lactic acid microspheres as subdermal fillers in animals
}

\author{
Chuan-Yuan Lin', Jui-Yu Lin', Dae-Yeol Yang ${ }^{2}$, Seong-Ho Lee ${ }^{3}$, Jeoung-Yong Kim ${ }^{4}$, Miyeon Kang ${ }^{5}$ \\ ${ }^{1}$ Tobeauty Aesthetic Clinic, Taipei 10582, Taiwan. \\ 2Department of Urology, Hallym University Dongtan Sacred Heart Hospital, Gyeonggi-do 18450, South Korea. \\ ${ }^{3}$ Department of Urology, Hallym University Kangdong Sacred Heart Hospital, Seoul 05355, South Korea. \\ ${ }^{4}$ Max Urology Clinic, Seoul 05051, South Korea. \\ ${ }^{5}$ R\&D center, Regen Biotech, Daejeon 34364, South Korea.
}

Correspondence to: Dr. Jeoung-Yong Kim, Max Urology, Jeil B/D 201, Achasan-ro 390, Gwangjin-gu, Seoul 05051, South Korea.

E-mail: aesthefill@aliyun.com

How to cite this article: Lin CY, Lin JY, Yang DY, Lee SH, Kim JY, Kang M. Efficacy and safety of poly-D,L-lactic acid microspheres as subdermal fillers in animals. Plast Aesthet Res 2019;6:16. http://dx.doi.org/10.20517/2347-9264.2019.23

Received: 17 Apr 2019 First Decision: 3 Jun 2019 Revised: 19 Jul 2019 Accepted: 20 Jul 2019 Published: 25 Jul 2019

Science Editor: Raúl González-García Copy Editor: Cai-Hong Wang Production Editor: Tian Zhang

\begin{abstract}
Aim: This animal study aims to examine the efficacy and safety of poly-D,L-lactic acid (PDLLA) microspheres as subdermal fillers.

Methods: Thirty 2-week-old male Sprague Dawley rats were used as test animals, and $0.5 \mathrm{~mL}$ filler solutions were injected into the subdermal tissues on their backs. Groups of five rats were randomly selected and sacrificed for examination on the 2nd, 4th, 8th, 12th, 16th, and 20th weeks after injection. Clinical and histological examinations were performed via the hematoxyline-eosin and immunohistochemical (IHC) staining of injected sites after collecting the injected masses. The body weights of the rats were measured, and the presence of filler substance in other organs was determined.
\end{abstract}

Results: Injected volumes were stable from the 2 nd to the 20th week after injection, and no abnormalities were observed around the injection sites. The injected substance did not migrate to the surrounding tissues. In IHC staining experiments, myofibroblasts were observed from the 2nd week, and collagen was detected from the 4th week. Myofibroblast was observed in the spaces between and inside the microspheres in the 8th week after injection, whereas type I collagen was found between and inside the microspheres at 8 th and 12th weeks, respectively.

Conclusion: The animal experiments confirm the efficacy and safety of injectable PDLLA as a subdermal filler.

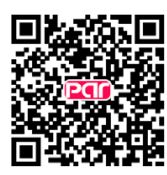


Keywords: Poly-D,L-lactic acid, injectables, fillers, microspheres, biostimulation

\section{INTRODUCTION}

Polylactic acid (PLA) was originally synthesized from $\alpha$-hydroxy acids by French chemists in 1954. This polymer has been used safely in resorbable suture materials, plates, and screws in orthopedic, neurologic, and craniofacial surgeries ${ }^{[1-6]}$. PLA forms the chiral molecules of poly-L-lactic acid (PLLA), poly-D-lactic acid, poly-D,L-lactic acid (PDLLA), and meso-PLA types ${ }^{[7]}$. Only PLLA and PDLLA have been extensively studied and have shown promising results ${ }^{[8-10]}$.

In 1999, injectable PLLA was approved for use in Europe (New-Fill; Biotech Industry SA, Luxembourg, Luxembourg). PLLA is used to increase the volumes of depressed skin areas, particularly to correct skin depressions such as creases, wrinkles, folds, scars, and eye rings ${ }^{[11]}$. PLLA is also useful for treating degenerative skin lesions due to aging. In August 2004, injectable PLLA (Sculptra; Dermik Laboratories, Bridgewater, NJ) was approved for the treatment of HIV-associated facial lipoatrophy in the United States ${ }^{[12]}$. In 2009 , this approval was expanded to include cosmetic applications ${ }^{[13]}$.

Injectable PDLLA is a new subdermal stimulatory filler (AestheFill; REGEN Biotech, Seoul, South Korea), and it has identical features as injectable PLLA. Injectable PDLLA is biocompatible, biodegradable, biostimulatory and long lasting. But the difference is the microparticles of injectable PDLLA are spongiform microspheres with multiple micropores. The aims of this study were to test the in vivo efficacy and safety of the injectable PDLLA as a subdermal tissue filler. This biodegradable polymer was injected into animals from September 1, 2009, to May 1, 2011. The effects and long-term utility of injected PDLLA microspheres were then investigated in observations of the dorsal parts of 2-week-old Sprague Dawley (SD) rats.

\section{MATERIALS AND METHODS}

\section{Materials}

\section{Biodegradable PDLLA filler}

The injectable PDLLA used in this study was produced by REGEN Biotech, and comprised 30 to $70 \mu \mathrm{m}$ PDLLA microspheres that were white, frozen, and dried solid. The microspheres were suspended in sodium carboxymethylcellulose as a carrier for injection.

\section{Experimental animals}

All animal procedures were conducted in compliance with the relevant laws and regulations of the Institutional Animal Care and Use Committee of Hallym University. A total of thirty 2-week old male SD rats were fed sufficient water and food in the animal facility of Hallym University.

\section{Methods}

Filler injection

$\mathrm{SD}$ rats were anesthetized with ketamine and xylazine and received subdermal injections of $0.5 \mathrm{~mL}$ PDLLA filler into their backs.

\section{Macroscopic observations of the injection sites}

On the 2 nd, 4 th, 8 th, 12 th, 16 th, and 20 th weeks after injection, five rats were randomly selected for clinical observations, and the skin color and volume changes at the injection sites were recorded. 


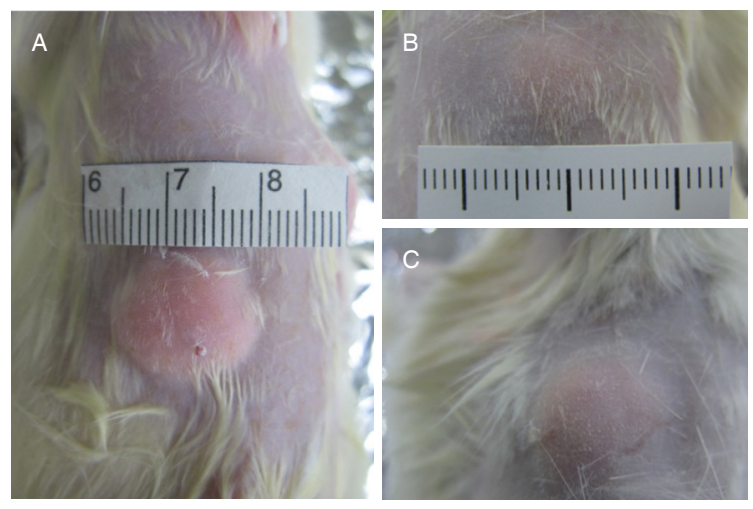

Figure 1. Macroscopic findings at injection sites. PDLLA filler injection sites can be visually confirmed. Inflammatory indicators, such as redness at the injection site or in the surrounding skin, were not present. A: immediately after PDLLA filler injection; B: at the 2nd week after PDLLA filler injection; C: at the 4th week after PDLLA filler injection. PDLLA: Poly-D,L-lactic acid
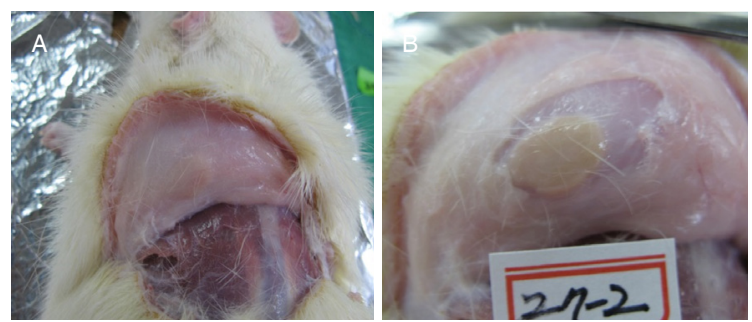

Figure 2. PDLLA filler injection sites at the 20th week. PDLLA filler was located in the subdermal tissue, and no abnormal findings, such as migration to surrounding tissues or inflammation, were apparent; A: skin reflection to show PDLLA filler mass; B: closer view of Figure 2A. PDLLA: Poly-D,L-lactic acid

\section{Collection and observation of tissues}

After the clinical observations of injection sites, tissues were harvested, immobilized in $10 \%$ neutral formalin solution, and then sliced into serial sections. Hematoxyline-eosin (H\&E) and immunohistochemical (IHC) staining analyses were performed using antibodies against collagen (type I) and actin. The degrees of proliferation of normal tissues were estimated on the basis of the observations of cells, myofibroblasts, and collagen.

\section{Safety of PDLLA filler}

The developmental states of all treated animals were monitored by periodically measuring their body weights. The transfer of the microspheres to other organs was validated histologically in the liver, kidney, spleen, and lung tissues from treated rats.

\section{RESULTS}

\section{Macroscopic findings}

After injecting PDLLA filler into the dorsal subdermal tissues of SD rats, no significant skin color changes were observed in the injection sites compared with the untreated sites, and circular elevations were noted [Figure 1A-C]. The PDLLA filler mass was visually pale yellow compared with the subdermal tissues from the inner side after peeling off the muscle tissues [Figure 2A and B]. PDLLA filler masses that formed circular shapes at the subdermal tissues of injection sites and PDLLA microspheres did not infiltrate into neighboring tissues during the 20th week of the study period after injection. Moreover, the injection volumes were maintained without significant reductions for the entire 20-week study period [Figure 3]. 


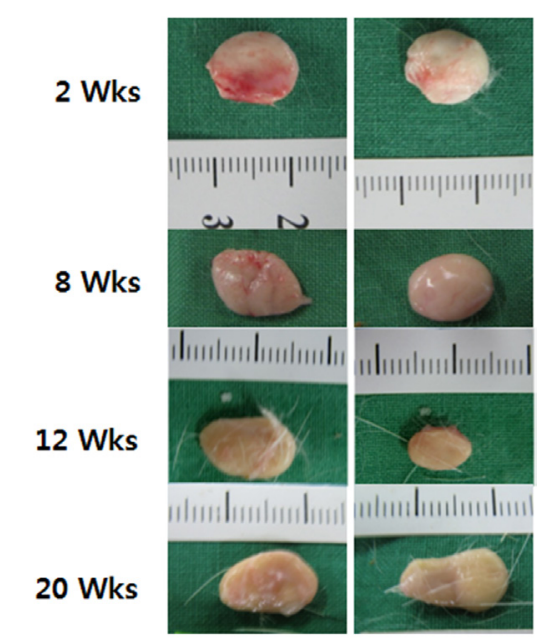

Figure 3. Changes in the sizes of poly-D,L-lactic acid filler masses over time. No significant reductions in volume were observed during the 20-week observation period after injection

\section{Histological findings}

Cell distributions in PDLLA filler masses

PDLLA filler masses were fixed in $10 \%$ neutral formalin solution, and serial sections were stained with H\&E. As shown in Figure 4A and B, PDLLA filler masses were localized to subdermal tissues and did not migrate to the surrounding tissues. Furthermore, although inflammatory cells were observed around PDLLA filler masses at the 2nd week after filler injections [Figure 4A], they tended to decrease at the 4 th week [Figure 4B].

In the analyses of serial sections, cells were observed in PDLLA filler masses. However, cell densities were not uniform throughout these masses at the 2nd and 8th weeks. Cell densities were low in the centers of filler masses early in the study period, particularly at the 2nd week. At the 12th and 20th weeks, cell densities were uniform throughout the filler masses. The high magnification $(\times 400)$ microscope observations show the foreign body giant cells in the spaces between and on the surfaces of the PDLLA microspheres at the 2 nd week [Figure 5A]. However, empty spaces remain between the microspheres. At the 8 th week [Figure 5B], these spaces were totally filled with giant cells; at the 12th and 20th weeks [Figure 5C and D], giant cells were visible in the spaces between the microspheres and within individual microspheres.

In Figure 6A, the H\&E stained section of an PDLLA filler mass at the 2nd week shows a vessel-like conduit. At the 12 th and 20 th weeks [Figure $6 \mathrm{~B}$ and $\mathrm{C}$ ], the vessel was increasingly evident and resembled a blood vessel.

New tissue formation (neotissue) in PDLLA filler masses

A. IHC staining for actin

To confirm the formation of neotissue in PDLLA filler masses, actin components were stained immunohistochemically in serial sections. Actin filaments are inside and the cytoskeleton of myofibroblasts. As shown in Figure 7A, myofibroblasts were visible around and in the spaces between the microspheres at the outer and inner parts of the mass from the 2nd week after PDLLA filler injections. At the 8th week, myofibroblasts were present in the entire mass and filled most of the spaces between the microspheres [Figure 7B]. At the 12th week, myofibroblasts were additionally present within individual microspheres [Figure 7C]; at the 20th week, myofibroblasts were further increased [Figure 7D]. 

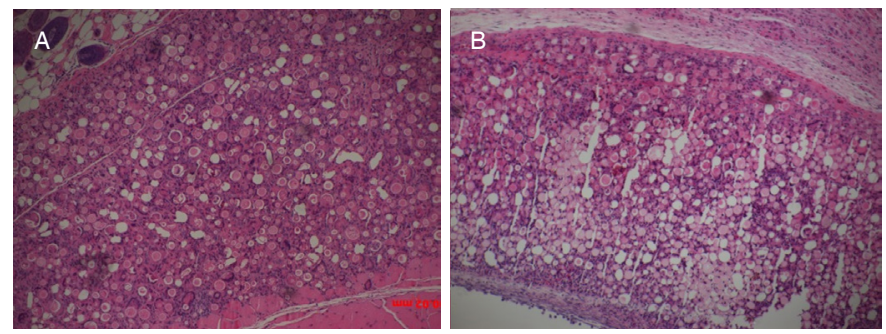

Figure 4. H\&E staining $(\times 100)$ after PDLLA filler injections. The injected PDLLA microspheres remained in the subdermal layer, and infiltration into surrounding tissues was not apparent. Inflammatory cells were visible around PDLLA microspheres. A: at the 2nd week; B: at the 4th week. PDLLA: poly-D,L-lactic acid

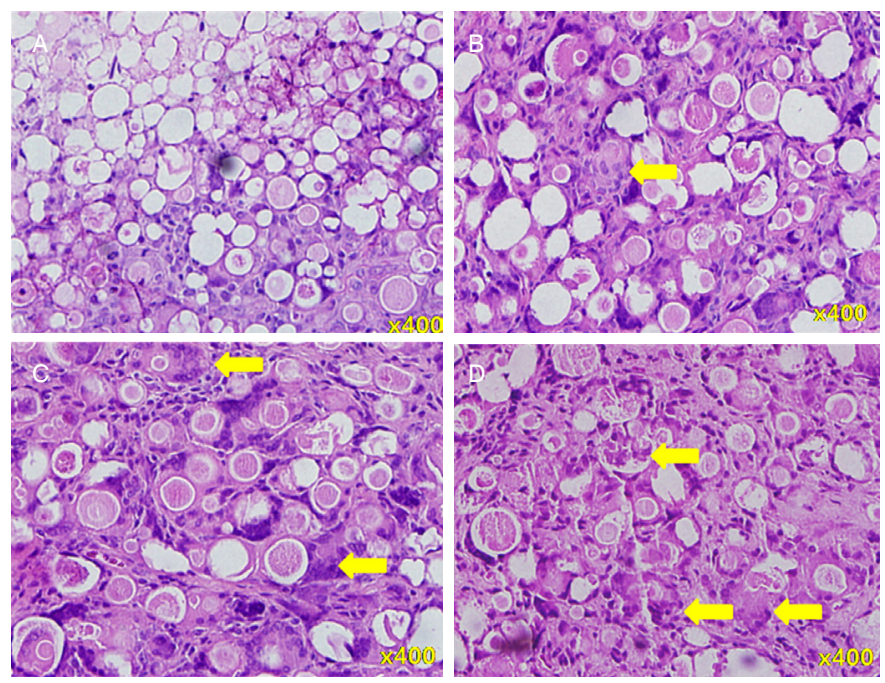

Figure 5. H\&E staining $(\times 400)$ at the 2 nd to 20 th week after poly-D,L-lactic acid filler injections. A: at the 2nd week; B: at the 8th week; C: at the 12th week; $D$ : at the 20th week. Yellow arrows: foreign body giant cells, with increasing number from $A$ to $D$
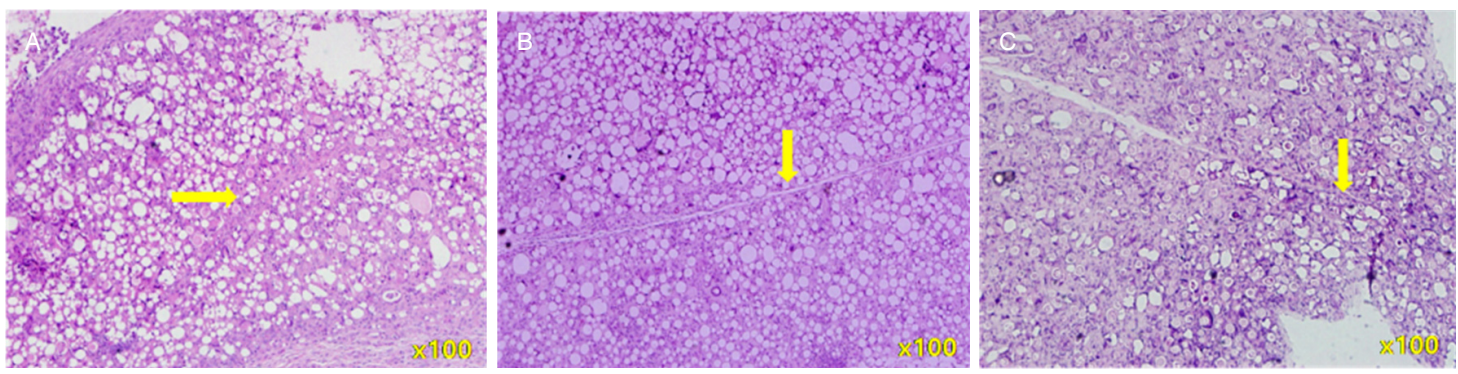

Figure 6. H\&E staining $(\times 100)$ pictures of an poly-D,L-lactic acid filler mass show a vessel-like conduit. A: at the 2nd week; B: at the 12th week; C: at the 20th week. Yellow arrows: vessel-like conduits

B. IHC staining for type I collagen

To confirm the formation of neotissue, we performed IHC analyses of filler mass sections by using antibodies against type I collagen. Unlike actin, collagen was expressed only in some parts between and on the surfaces of the microspheres at the 4 th week [Figure $8 \mathrm{~A}$ ]. At the 8 th week, the collagen expression between the microspheres was greater than that at the 4 th week [Figure $8 \mathrm{~B}$ ].

At the 12th week after PDLLA filler injection, the appearance of collagen was increased [Figure $8 \mathrm{C}$ ]. As indicated by the arrows, collagen was present inside the individual microspheres. Moreover, at the 20th week, collagen expression was increased inside the individual microspheres [Figure 8D]. 


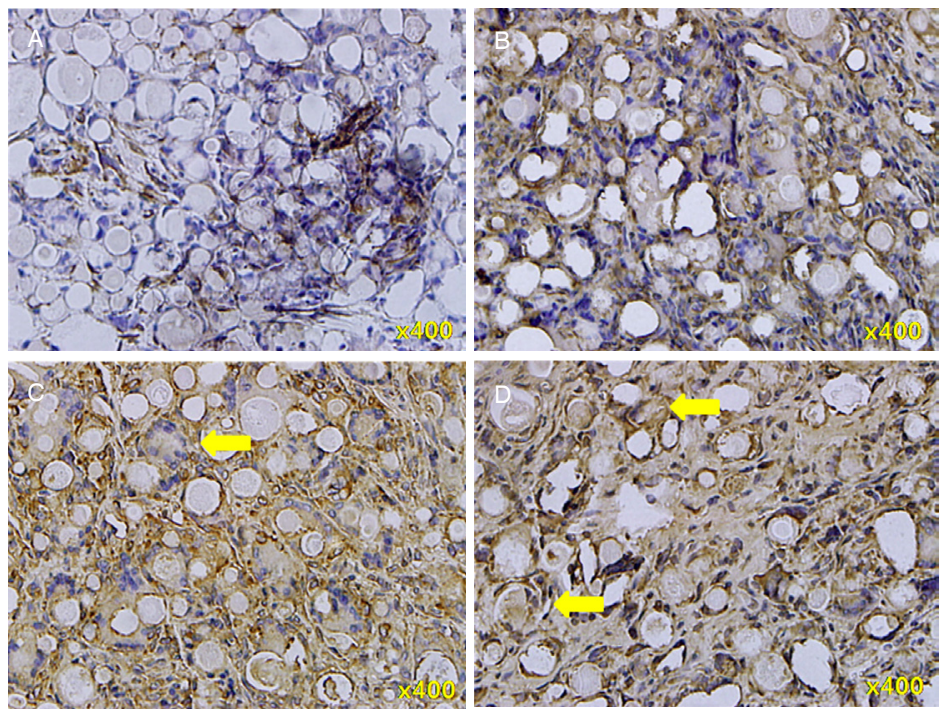

Figure 7. Immunohistochemical staining of actin $(\times 400)$ in PDLLA filler mass sections at the 2 nd to 20th weeks after PDLLA filler injections. A: at the 2nd week; B: at the 8th week; C: at the 12th week; D: at the 20th week. Actin filaments are inside and the cytoskeleton of myofibroblasts. Yellow arrows: myofibroblasts, with increasing number from A to D. PDLLA: poly-D,L-lactic acid

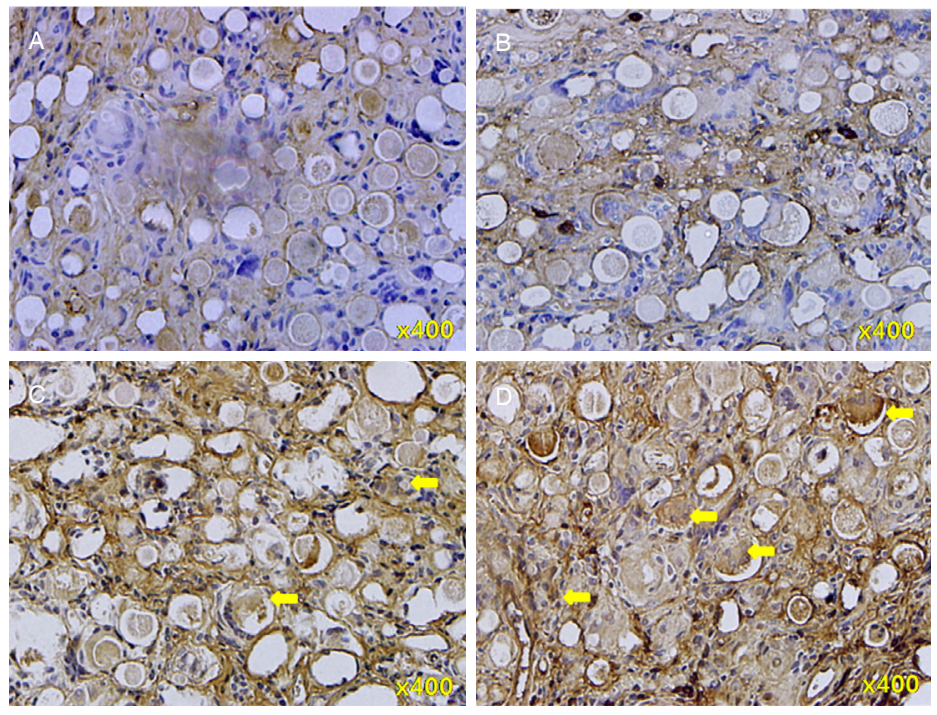

Figure 8. Immunohistochemical staining of type I collagen in neotissues $(\times 400)$ at the 4 th to 20 th weeks after poly-D,L-lactic acid filler injections. A: at the 4th week; B: at the 8th week; C: at the 12th week; D: at the 20th week. Yellow arrows: type I collagen, with increasing number from $A$ to $D$

\section{Changes in body weight}

No significant weight gains or losses were observed after the injection of the filler into experimental animals.

\section{Transfer of PDLLA microspheres to other organs}

After PDLLA filler was injected, liver, kidney, spleen, and lung tissues were harvested and fixed in 10\% neutral formalin solution. H\&E staining was then performed with serial sections. These analyses show that the microspheres did not migrate into distant organs [Figure 9A-D]. 

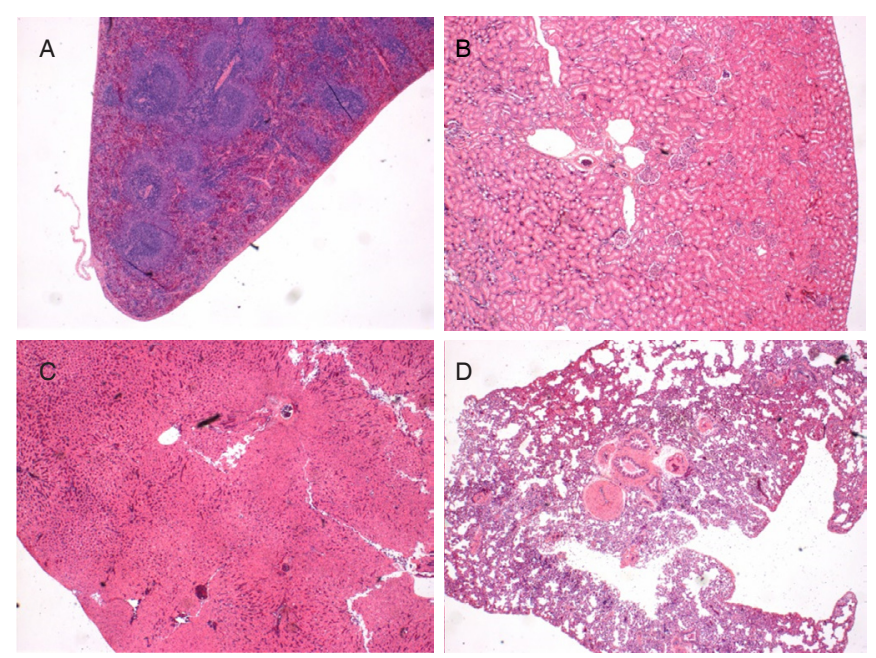

Figure 9. The migration of PDLLA microspheres to internal organs was not observed after PDLLA filler injections. A: lung; B: spleen; C: liver; D: kidney. PDLLA: poly-D,L-lactic acid

\section{DISCUSSION}

Injectable fillers offer simple and minimally invasive techniques for tissue volume expansion. Ideal materials for such interventions must be easily injectable, nonmigratory, noninflammatory, volume stable, biodegradable, and biocompatible. Many types of tissue fillers are currently used for cosmetic and medical indications in routine clinical practice ${ }^{[14]}$. These tissue fillers can be classified as temporary, semipermanent, or permanent fillers depending on the duration of the injected product in tissues ${ }^{[15]}$. These fillers are also classified according to their compositions, and collagen (bovine, porcine, and human), hyaluronic acid (HA), PLLA, calcium hydroxylapatite (CaHA), polymethyl methacrylate, polyacrylamide, and autologous fat cells have been investigated ${ }^{[15]}$. Among these fillers, CaHA and PLLA are known as collagen stimulators $^{[16]}$ and offer unique and effective ways to address tissue impediments with natural-appearing results and durability.

Poly-lactic-co-glycolic acid (PLGA) microspheres have been considered injectable bulking substances in previous studies ${ }^{[17-19]}$. These studies show that PLGA is biodegradable and biocompatible and induces hybrid tissue formation upon implantation. However, PLGA microspheres fail to offer a long-term (more than six months) maintenance of hybrid tissue volumes because they degrade. Compared with PLGA, PLA microspheres have slower degradation rates owing to their relative hydrophobicity. Hence, injectable PLA microspheres maintain hybrid tissue volumes for longer periods. Kang et al. ${ }^{[20]}$ previously showed that volumes of implanted PLA in dorsum tissues of mice slowly decreased in volume to $52 \%$ after 12 months but maintained this residual volume until 18 months.

In this study, PDLLA filler affected the formation of neotissues around and inside injection areas, similar to PLLA demonstrations as a collagen stimulator. Moreover, during the test period, no clinical symptoms or changes in body weights were observed in our experimental animals. No migration of test substance to the lung, spleen, liver, or kidney tissues was observed, and the injected PDLLA filler volumes in the sub-dermis were maintained between the 2nd and 20th weeks after injection. We also observed no abnormal findings, such as inflammation around and inside the injection sites. Histopathological findings similarly showed the appropriate localization of injected PDLLA filler in subdermal tissues and confirmed that the substance did not migrate to surrounding tissues.

Cells that were distributed in the periphery of PDLLA filler injection sites moved into the spaces between PDLLA microspheres and then moved into the centers of the microspheres. Nutrient supply through 
microvessels is necessary for the proliferation and migration of cells into injected masses. As shown in Figure 5A-D, the spaces between PDLLA microspheres filled with cells over time, thus suggesting the formation of blood vessel-like pathways that may supply nutrients for cell proliferation inside the PDLLA filler mass.

To investigate the formation of neotissues, we performed IHC analyses on the actin and type I collagen in sections of the PDLLA filler masses. Myofibroblasts were found between the microspheres as early as two weeks after injection and continued to develop and eventually penetrated the PDLLA microspheres. Similarly, extracellular type I collagen was detected in spaces between and on the outer surfaces of the microspheres at the 4 th week and was found inside the individual microspheres by the 20 th week.

This animal study suggests that injected PDLLA filler mass maintains its volume by facilitating the formation of new tissues, which ultimately replace the volume of the PDLLA filler mass and presumably hydrolyze the PDLLA.

We can divide the volume maintenance into two stages. The first stage of the volume increase is from the volume of the injected PDLLA microspheres. The second stage is volume maintenance, and it is due to the formation of new tissues and collagen with cell inflow between and inside PDLLA microspheres. The volume of new tissues and collagen replaces the volume of PDLLA microspheres, which are eventually hydrolyzed.

In 2012, a clinical study was performed to determine the efficacy and safety of PDLLA filler injections for penile augmentation ${ }^{[21]}$. The significant penile augmentation effect lasted for 18 months after injection and was well tolerated without serious adverse effects. Subsequently, 58 people were recruited into a randomized, evaluator-blinded, comparative study that was conducted on August 1, 2012 to March 6, 2013. In this study, the efficacy and safety of PDLLA microspheres injections for the correction of nasolabial folds were compared with those of HA. PDLLA microspheres were not inferior to $\mathrm{HA}^{[22]}$, and 30 subjects completed the 24-month long-term safety evaluation follow up, which showed that PDLLA microspheres are safe and effective for use as fillers for nasolabial fold correction ${ }^{[23]}$. Complications such as granuloma formation $^{[24-27]}$ and accidental vascular occlusion ${ }^{[26-28]}$ could occur, similar to other subdermal fillers, and need to be investigated further.

In this study, rat body weights did not change during the experimental period, and no evidence of test substance migration to other organs was found. The injected volumes were maintained for 20 weeks in part because of the inflow and growth of cells, actin, and type I collagen inside the microspheres. The formation of neotissues that replace the original volume of the PDLLA filler mass was further suggested by observations of microvessels inside PDLLA filler masses. Therefore, injectable PDLLA polymer is biodegradable and has efficacy and safety as a subdermal tissue filler.

\section{DECLARATIONS}

\section{Acknowledgments}

The authors want to give special thanks to Dr. Lee Suat-Yee, a pathologist of Chung Shan Medical University Hospital, Taichung, Taiwan, for her kindly help of reviewing for all the histology results. The authors also thank Enago - https://www.enago.tw/ for their assistance in manuscript editing.

\section{Authors' contributions}

Concept and design: Kim JY

Data acquisition, technical and material support: Kang M

Data analysis, manuscript preparation: Yang DY, Lee SH

Critical revision and completion of manuscript: Lin CY, Lin JY 


\section{Availability of data and materials}

Not applicable.

\section{Financial support and sponsorship}

This work was supported by Regen Biotech, Inc., Seoul, South Korea.

\section{Conflicts of interest}

Dr. Dae-Yeol Yang has no conflicts to disclose. Dr. Chuan-Yuan Lin is medical director for Regen. Dr. JuiYu Lin is chief medical director for Regen. Dr. Seong-Ho Lee has no conflicts to disclose. Miyeon Kang is director for Regen R\&D center. Dr. Jeoung-Yong Kim is chief director for Regen R\&D center.

\section{Ethical approval and consent to participate}

The study has been approved by relevant ethical committee and the whole research process complied with ethical guidelines.

\section{Consent for publication}

Not applicable.

\section{Copyright}

(c) The Author(s) 2019.

\section{REFERENCES}

1. Middleton JC, Tipton AJ. Synthetic biodegradable polymers as orthopedic devices. Biomaterials 2000;21:2335-46.

2. Kulkarni RK, Pani KC, Neuman C, Leonard F. Polylactic acid for surgical implants. Arch Surg 1966;93:839-43.

3. Achtnich A, Forkel P, Metzlaff S, Zantop T, Petersen W. Degradation of poly-D-L-lactide (PDLLA) interference screws (Megafix (R)). Arch Orthop Trauma Surg 2014;134:1147-53.

4. Farah S, Anderson DG, Langer R. Physical and mechanical properties of PLA, and their functions in widespread applications - a comprehensive review. Adv Drug Deliv Rev 2016 15;107:367-92.

5. Saini P, Arora M, Kumar M. Poly(lactic acid) blends in biomedical applications. Adv Drug Deliv Rev 2016;107:47-59.

6. Athanasiou KA, Niederauer GG, Agrawal CM. Sterilization, toxicity, biocompatibility and clinical applications of polylactic acid/ polyglycolic acid copolymers. Biomaterials 1996;17:93-102.

7. Pretula J, Slomkowski S, Penczek S. Polylactides-Methods of synthesis and characterization. Adv Drug Deliv Rev 2016;107:3-16.

8. Grandfils C, Flandroy P, Nihant N, Barbette S, Jerome R, et al. Preparation of poly (D,L) lactide microspheres by emulsion-solvent evaporation, and their clinical applications as a convenient embolic material. J Biomed Mater Res 1992;26:467-79.

9. Robinson BP, Hollinger JO, Szachowicz EH, Brekke J. Calvarial bone repair with porous D,L-polylactide. Otolaryngol Head Neck Surg 1995;112:707-13.

10. Sherwood JK, Riley SL, Palazzolo R, Brown SC, Monkhouse DC, et al. A three-dimensional osteochondral composite scaffold for articular cartilage repair. Biomaterials 2002;23:4739-51.

11. Valantin MA, Aubron-Olivier C, Ghosn J, Laglenne E, Pauchard M, et al. Polylactic acid implants (New-Fill) to correct facial lipoatrophy in HIV-infected patients: results of the open-label study VEGA. AIDS 2003;17:2471-7.

12. Humble G, Mest D. Soft tissue augmentation using sculptra. Facial Plast Surg 2004;20:157-63.

13. Palm MD, Woodhall KE, Butterwick KJ, Goldman MP. Cosmetic use of poly-1-lactic acid: a retrospective study of 130 patients. Dermatol Surg 2010;36:161-70.

14. Attenello NH, Maas CS. Injectable fillers: review of material and properties. Facial Plast Surg 2015;31:29-34.

15. Sanchez-Carpintero I, Candelas D, Ruiz-Rodriguez R. Dermal fillers: types, indications, and complications. Actas Dermosifiliogr 2010;101:381-93.

16. Breithaupt A, Fitzgerald R. Collagen stimulators: poly-L-Lactic acid and calcium hydroxyl apatite. Facial Plast Surg Clin North Am 2015;23:459-69.

17. Cho ER, Kang SW, Kim BS. Poly(lactic-co-glycolic acid) microspheres as a potential bulking agent for urological injection therapy: preliminary results. J Biomed Mater Res B Appl Biomater 2005;72:166-72.

18. Kang SW, Cho ER, Kim BS. PLGA microspheres in hyaluronic acid gel as a potential bulking agent for urologic and dermatologic injection therapies. J Microbiol Biotechnol 2005;15:510-8.

19. Cho ER, Kang SW, Park HJ, Cho YS, Lee YS, et al. Submucosal injection of poly(lactic-co-glycolic acid) microspheres in rabbit bladder as a potential treatment for urinary incontinence and vesicoureteral reflux: preliminary results. J Biomater Sci Polym Ed 2005; 16:1109-20.

20. Kang SW, Cho ER, Jeon O, Kim BS. The effect of microsphere degradation rate on the efficacy of polymeric microspheres as bulking 
agents: an 18-month follow-up study. J Biomed Mater Res B Appl Biomater 2007;80:253-9.

21. Yang DY, Ko K, Lee SH, Moon DG, Kim JW, et al. Efficacy and safety of a newly developed polylactic acid microsphere as an injectable bulking agent for penile augmentation: 18-months follow-up. Int J Impot Res 2017;29:136-41.

22. Hyun MY, Lee Y, No YA, Yoo KH, Kim MN, et al. Efficacy and safety of injection with poly-L-lactic acid compared with hyaluronic acid for correction of nasolabial fold: a randomized, evaluator-blinded, comparative study. Clin Exp Dermatol 2015;40:129-35.

23. No YA, Seok J, Hyun MY, Kwon TR, Oh CT, et al. Long-term (24-month) safety evaluation of poly-DL-lactic acid filler injection for the nasolabial fold: a multicenter, open, randomized, evaluator-blind, active-controlled design. Plast Reconstr Surg 2015;135:1074-5.

24. Lee JM, Kim YJ. Foreign body granulomas after the use of dermal fillers: pathophysiology, clinical appearance, histologic features, and treatment. Arch Plast Surg 2015;42:232-39.

25. Molina-Ruiz AM, Requena L. Foreign body granulomas. Dermatol Clin 2015;33:497-523.

26. Woodward J, Khan T, Martin J. Facial filler complications. Facial Plast Surg Clin North Am 2015;23:447-58.

27. Urdiales-Galvez F, Delgado NE, Figueiredo V, Lajo-Plaza JV, Mira M, et al. Treatment of soft tissue filler complications: Expert consensus recommendations. Aesthetic Plast Surg 2018;42:498-510.

28. Ansari ZA, Choi CJ, Rong AJ, Erickson BP, Tse DT. Ocular and cerebral infarction from periocular filler injection. Orbit 2018;30:1-3. 